\title{
Electromagnetically driven convection suitable for mass transfer enhancement in liquid metal batteries
}

\author{
Norbert Weber ${ }^{\mathrm{a}}$, Michael Nimtz ${ }^{\mathrm{a}}$, Paolo Personnettaz ${ }^{\mathrm{a}, \mathrm{c}}$, Alejandro Salas ${ }^{\mathrm{a}, \mathrm{b}}$, \\ Tom Weier ${ }^{\mathrm{a}}$ \\ ${ }^{a}$ Helmholtz-Zentrum Dresden - Rossendorf, Bautzner Landstr. 400, Dresden, Germany \\ ${ }^{b}$ Instituto Tecnológico y de Estudios Superiores de Monterrey, Monterrey, Mexico \\ ${ }^{c}$ Politecnico di Torino, Corso Duca degli Abruzzi 24, 10129 Torino, Italy
}

\begin{abstract}
Liquid metal batteries (LMBs) were recently proposed as cheap large scale energy storage. Such devices are urgently required for balancing highly fluctuating renewable energy sources. During discharge, intermetallic phases tend to form in the cathode of LMBs. These do not only limit the up-scalability, but also the efficiency of the cells. Generating a mild fluid flow in the fully liquid cell will smoothen concentration gradients and minimise the formation of intermetallics. In this context we study electro-vortex flow numerically. We simulate a recent LMB related experiment and discuss how the feeding lines to the cell can be optimised to enhance mass transfer. The Lorentz forces have to overcome the stable thermal stratification in the cathode of the cell; we show that thermal effects may reduce electro-vortex flow velocities considerable. Finally, we study the influence of the Earth magnetic field on the flow.

Keywords: liquid metal battery, electro-vortex flow, mass transfer enhancement, swirl, Rayleigh-Bénard convection, OpenFOAM, thermal stratification
\end{abstract}




\section{Introduction}

Integrating highly fluctuating renewable energy sources (such as photovoltaics and wind power) into the electric grid calls for large scale energy storage. Such storage must be, first of all, safe and cheap. The liquid metal battery (LMB) promises both. After being intensively investigated in the 1960s, and abandoned later, LMB research experienced a renaissance some ten years ago. For an overview of the pioneering work, see [1] (recommended [4) and for the recent work [5] and [6].
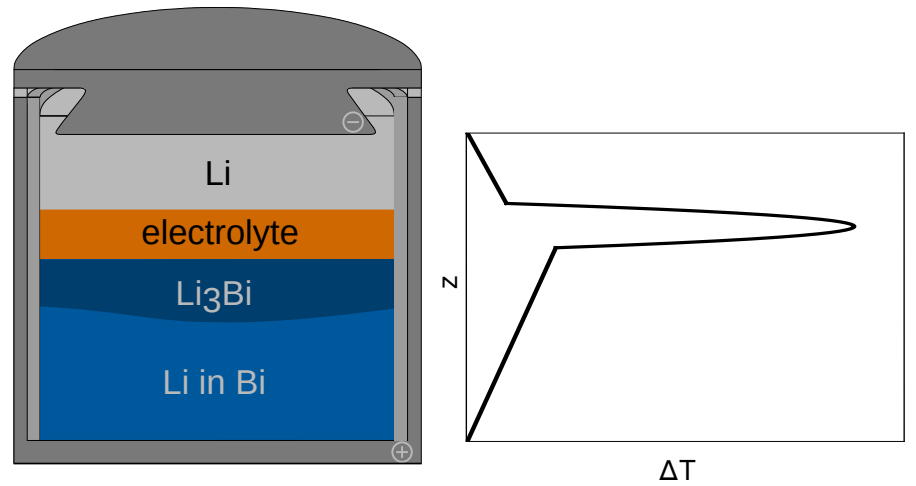

Figure 1: Sketch of a typical Li||Bi liquid metal battery with an intermetallic phase forming in the cathode (left) and vertical temperature distribution in the three layers for pure diffusion (right).

Fig. 17a shows a sketch of a typical LMB. A dense metal on the bottom (cathode, positive electrode) is separated by a liquid salt from a lighter metal at the top (anode, negative electrode). All three phases float above each other; the salt acts as the electrolyte. The word "liquid metal battery" names only a family of electrochemical cells (which may consists of many different active metals combinations). Typical cell couples include $\mathrm{Ca} \| \mathrm{Bi}$ 7, 8], $\mathrm{Ca} \| \mathrm{Pb}[9]$, $\mathrm{K}|| \mathrm{Hg}$ [10, 11, $\mathrm{Li}|| \mathrm{Bi}$ 1, 12 16, Li || $\mathrm{Pb}$ 1, 17, $\mathrm{Li}|| \mathrm{Sb}$ [5, 17, $\mathrm{Li}|| \mathrm{Sn}$ 1, 14, 1820, $\mathrm{Li}|| \mathrm{Zn}$ 1], Mg||Sb [5, 21, 22], Na||Bi [1, 14, 18, 20, 23, 27], Na||Hg [5, 28, 29, $\mathrm{Na}|| \mathrm{Pb}$ [1, 14, 20, 27, 30, $\mathrm{Na}|| \mathrm{Sn}[1,11,18,20,31,32$ and $\mathrm{Na}|| \mathrm{Zn}$ [33, 34] as well as exotic ones such as Li||Se [1, 35, 36] or Li || $\mathrm{Te}$ [1, 14, 15, 35, 36]. 
During discharge, the anode metal is oxidised, crosses the electrolyte layer and alloys in the bottom layer with the dense metal ("concentration cell"). Commonly, the ohmic resistance of the electrolyte layer represents the most important overvoltage. However, at higher discharge currents concentration polarisation enters the field [5, 11, 22, 28, 32, 37]. Example: when discharging a Li||Bi cell, Li-rich alloy will concentrate at the cathode-electrolyte interface. When a certain local concentration is exceeded, a solid intermetallic phase $\left(\mathrm{Li}_{3} \mathrm{Bi}\right)$ will form (fig. 17) 1, 24]. Such intermetallics often float on the cathode metal 38]. Sometimes they expand during solidification. As the walls impede a lateral expansion, the intermetallic will form a dome until finally short-circuiting the electrolyte. Especially in Ca based cells, locally growing dendrites may additionally short-circuit the cell [7]. Besides of all the mentioned drawbacks, the formation of intermetallics has one advantage: it removes anode metal from the melt and keeps thereby the voltage constant. It should be also mentioned that some intermetallics have high electrical resistances while others are good conductors.

When charging the cell of fig. 1 1a, the positive electrode-electrolyte interface will deplete of $\mathrm{Li}$ and a similar concentration gradient may develop 24]. This effect is undesirable, too. Finally, all the same effects may theoretically happen in the anode compartment, too, if an alloyed top electrode is used (e.g. Ca-Mg [8, 21]). However, such effects were not reported, yet.

It was early proposed that a mild fluid flow may counterbalance concentration gradients and increase thereby the efficiency of LMBs [1, 24, 37]. While "mechanical stirring" [1, 37] seems difficult to realise, a localised heating or cooling inducing thermal convection may be a very good option [39, 40. Electrovortex flow (EVF) may be used for an efficient mass transfer enhancement, too [4143. Simply saying, EVF always may develop when current lines are not in parallel. It can therefore easily be adjusted by choosing the diameter/geometry of the current collectors and feeding lines appropriately. EVF drives a jet away from the wall, forming a poloidal flow [4]. For a classical example of the origin of EVF, see Lundquist [4] and Shercliff [45, for a good introduction Davidson 
[46] and a detailed overview including many experiments Bojarevics et al. 47]. The relevance of EVF for LMBs is outlined by Ashour et al. [43]. It should also be mentioned that other flow phenomena like the Tayler instability [48[56], Rayleigh-Bénard convection [57, 58] or interface instabilities [59 63] may enhance mass transfer in LMBs, as well.

This article is dedicated (mainly) to electro-vortex flow. It's aim is twofold: first, we will show how the connection of the supply lines to the cell influences the flow. Second, we study how electro-vortex flow and stable thermal stratification interact. For this purpose we combine numerical simulation with a simple 1D heat conduction model. These models - and the experiment which inspired our studies - are described in the following section.

\section{Physical, mathematical and numerical model}

In this section we will first present the experiment 64] which inspired this article. Thereafter we explain the way in which we estimate the temperature gradient appearing in the cathode of a liquid metal battery (LMB). Finally, we give an introduction to the 3D numerical models used.

\subsection{Liquid metal electrode experiment}

Fig. 21llustrates the mentioned experiment, conducted by Kelley \& Sadoway 64]. A cylindrical steel vessel contained a melt of eutectic lead-bismuth. An

electric current (up to $0.375 \mathrm{~A} / \mathrm{cm}^{2}$ ) was applied between a bottom and top electrode. The bottom current was supplied centrically or laterally. The upper electrode consisted of a nickel-iron foam; such foam is often used in LMBs to contain the anode metal [6]. As the setup was heated from below, RayleighBénard cells appear. If an internal current was applied, the flow became much more regular at $0.05 \mathrm{~A} / \mathrm{cm}^{2}$. It was deduced by the authors that convection cells align with the magnetic field. We will demonstrate how electro-vortex flow may give an alternative explanation for the increase in order.

We use the following material properties of lead bismuth eutectic (LBE) at $160^{\circ} \mathrm{C}$ [3] : a kinematic viscosity of $\nu=2.7 \cdot 10^{-7} \mathrm{~m}^{2} / \mathrm{s}$, a thermal expansion 


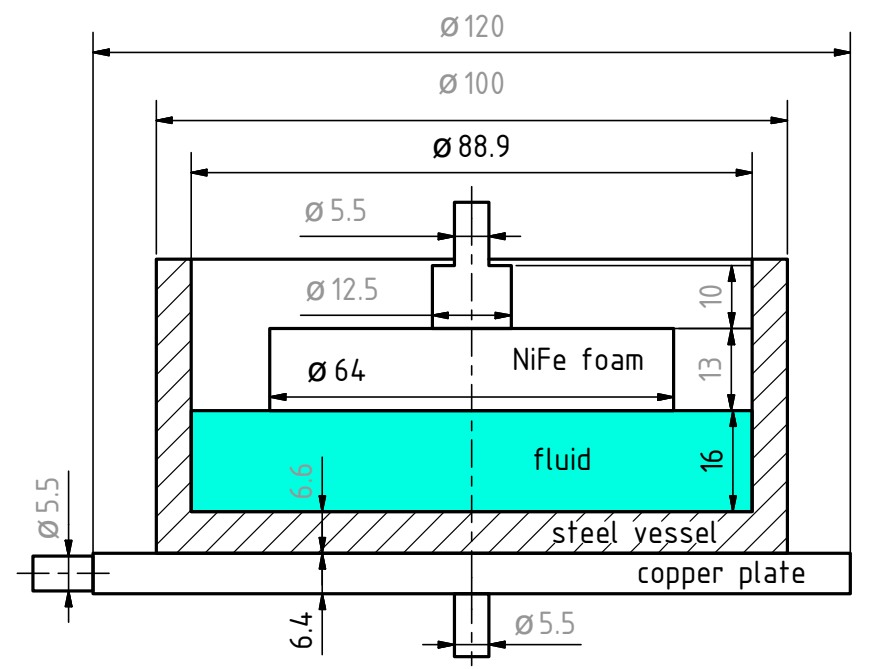

Figure 2: Dimensions of the experiment and simulation model (in $\mathrm{mm}$ ). The gray values are not exactly known; they are estimated from the sketch in 64]. The wires are assumed to be made of copper.

coefficient of $\beta=1.3 \cdot 10^{-4} \mathrm{~K}^{-1}$, an electrical conductivity of $\sigma=9 \cdot 10^{5} \mathrm{~S} / \mathrm{m}$, a density of $\rho=10505 \mathrm{~kg} / \mathrm{m}^{3}$, a specific heat capacity of $c_{p}=148 \mathrm{~J} / \mathrm{kg} / \mathrm{K}$, a thermal conductivity of $\lambda=10 \mathrm{~W} / \mathrm{m} / \mathrm{K}$, a thermal diffusivity of $\alpha=6 \cdot 10^{-6} \mathrm{~m}^{2} / \mathrm{s}$, a Prandtl number of $\operatorname{Pr}=0.04$ and a sound velocity of $u_{\mathrm{s}}=1765 \mathrm{~m} / \mathrm{s} 6567$. The electrical conductivity of the vessel is assumed to be $\sigma=1.37 \cdot 10^{6} \mathrm{~S} / \mathrm{m}$ and of the wires and copper plate $\sigma=58.1 \cdot 10^{7} \mathrm{~S} / \mathrm{m}$. The electrical conductivity of the Fe-Ni foam is not easy to determine [68, 69], especially because it is not sure if the liquid metal enteres the pores. We do not model the porosity and use an electric conductivity of $\sigma=1.37 \cdot 10^{6} \mathrm{~S} / \mathrm{m}$ without further justification.

Geometrically, the described experiment perfectly represents a liquid cathode of an LMB. However, the temperature gradient in a working LMB depends on the boundary conditions. For a single cell with insulated lateral walls it will rather be opposite to that in the experiment. As the electrolyte layer has the highest electrical resistance (four orders larger than the metals), most heat will be generated there [57. Fig. 10 shows a typical vertical temperature profile through all three layers. If no thermal management system induces additional 


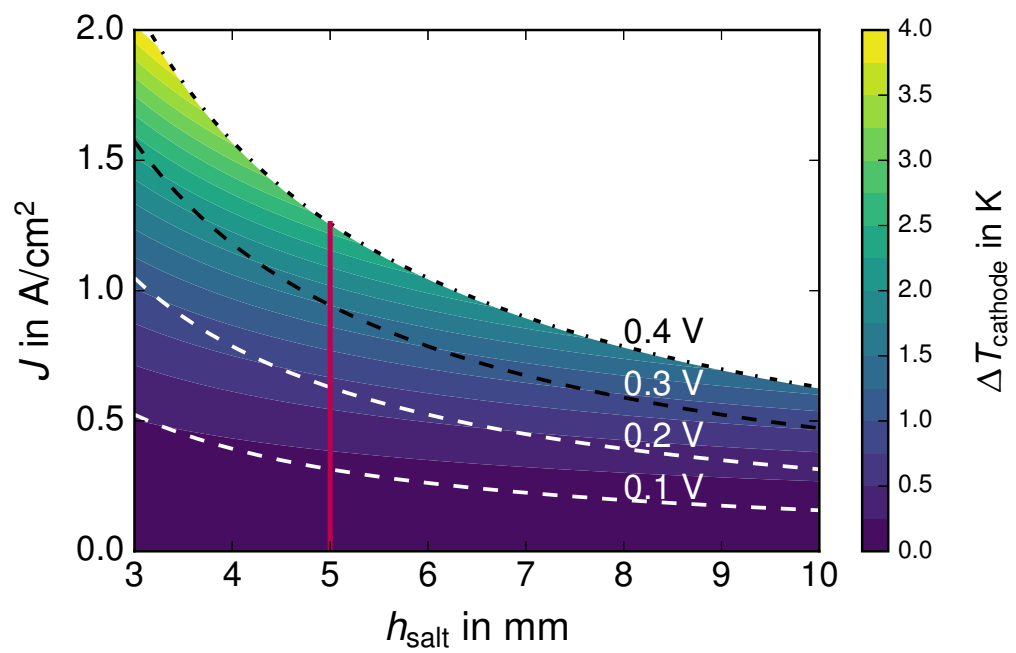

Figure 3: Voltage drop and temperature difference in the cathode for pure conduction in a Li||Bi cell [70].

temperature gradients (as suggested in [21, 40]) a stable thermal stratification is expected in the cathode. We will study here, if electro-vortex flow can overcome this stratification. For this purpose, we need a characteristic vertical temperature gradient. As no LMB exists, which operates at such low temperature as the experiment, we use a similar cell to define a typical vertical temperature gradient: a $\mathrm{Li}|| \mathrm{Bi} \mathrm{LMB}$ operating at $450^{\circ} \mathrm{C}$.

\subsection{Heat conduction model}

The temperature difference between top and bottom of the cathode of an LMB can be estimated using the simple 1D heat conduction model developed by Personnettaz [70] (for 3D studies of heat transfer in Li-LMBs, see [58, 71]).

The model was developed with the assumption of a fluid at rest, constant and homogeneous material properties and a uniform current density distribution. The heat generation was included only in the form of Joule heating in the electrolyte layer, due to its high electrical resistivity $\left(\frac{\rho_{\mathrm{el}, \mathrm{salt}}}{\rho_{\mathrm{el}, \mathrm{metal}}}>10^{3}\right)$. The lateral wall of the cell was considered adiabatic and the top and the bottom 
boundaries are set to a constant temperature $T=450{ }^{\circ} \mathrm{C}$. Thanks to the mentioned hypotheses a 1D model along the vertical coordinate is able to completely describe the temperature distribution inside the cell. This profile provides an upper bound for the temperature and a valuable initial guess of the temperature stratification in the cathode. The cell studied by Personnettaz was a Li|KCl$\mathrm{LiCl} \mid \mathrm{Bi} \mathrm{LMB}$ [70]. We use his model to estimate the temperature difference between the top and bottom of the cathode as

$$
\Delta T=\frac{h_{\mathrm{Bi}} h_{\text {salt }}\left(2 h_{\mathrm{Li}} \lambda_{\text {salt }}+h_{\text {salt }} \lambda_{\mathrm{Li}}\right) \rho_{\mathrm{el}, \mathrm{salt}} J^{2}}{2 h_{\mathrm{Bi}} \lambda_{\mathrm{Li}} \lambda_{\text {salt }}+2 h_{\mathrm{Li}} \lambda_{\mathrm{Bi}} \lambda_{\text {salt }}+2 h_{\text {salt }} \lambda_{\mathrm{Bi}} \lambda_{\mathrm{Li}}}
$$

with $h, \lambda, \rho_{\mathrm{el}, \text { salt }}$ and $J$ denoting the layer heights, the thermal conductivities, the specific resistance of the salt and the current density. The thickness of the cathode is set to $16 \mathrm{~mm}$ as in the experiment and the Li-layer to $32 \mathrm{~mm}$ in order to maximise the cell capacity (see [70]). The geometrical parameters and the transport properties employed are collected in tab. 1. Depending on the current density $J$ and thickness of the electrolyte $h_{\text {salt }}, \Delta T$ over the cathode changes as illustrated in fig. 3 .

We limit the maximum ohmic over-voltage to $0.4 \mathrm{~V}$ which corresponds to a cell efficiency of about $66 \%$. We assume further that the electrolyte is $5 \mathrm{~mm}$ thick (realistic values are $3-15 \mathrm{~mm}$ [72]) and find the following law that describes the dependence of the temperature difference by the current $I$ (assuming a cathode base area of $62.1 \mathrm{~cm}^{2}$ ) as

$$
\Delta T(\mathrm{~K})=4.37 \times 10^{-4}\left(\mathrm{~K} / \mathrm{A}^{2}\right) \cdot I^{2}\left(\mathrm{~A}^{2}\right) .
$$

This formula provides only a rough estimate of a possible temperature difference in the cathode. The multiplicative factor strongly depends on the materials selection and their transport properties at the operation temperature. Anyway it provides a first estimate that allows to study the competition of two current driven phenomena, electro-vortex flow and thermal stratification, in a low temperature liquid metal experiment. 
Table 1: Properties of the pure substances at $T=450^{\circ} \mathrm{C}$ and dimension for the pure heat conduction model [73-77]. The molten salt employed is $\mathrm{KCl}-\mathrm{LiCl}$.

\begin{tabular}{llrrr}
\hline property & \multicolumn{1}{c}{ unit } & $\mathrm{Li}$ & salt & $\mathrm{Bi}$ \\
\hline$h_{i}$ & $\mathrm{~mm}$ & 32 & 5 & 16 \\
\hline$k$ & $\mathrm{~W} /(\mathrm{m} \mathrm{K})$ & 53.0 & 0.69 & 14.2 \\
$\sigma_{\text {el }}$ & $\mathrm{S} / \mathrm{m}$ & - & 157 & - \\
$\rho_{\text {el }}$ & $10^{-2} \Omega \mathrm{m}$ & - & 6.36 & - \\
\hline
\end{tabular}

\subsection{Numerical model}

The numerical model is implemented in OpenFOAM [78]; for all details and the validation of the electro-vortex flow solver see [79. Basically, it computes the electric potential $\phi$ and current density $\boldsymbol{J}$ on a global mesh as

$$
\begin{aligned}
\nabla \cdot \sigma \nabla \phi & =0 \\
\boldsymbol{J} & =-\sigma \nabla \phi
\end{aligned}
$$

with $\sigma$ denoting the electrical conductivity. All conducting regions (of different conductivities - see fig. 3) are fully coupled. The results are then mapped on a separate fluid mesh (blue area in fig. 3). Induced currents and magnetic fields are neglected, which is justified as long as the velocities are small. On the fluid mesh the following set of equations is solved:

$$
\begin{aligned}
& \frac{\partial \boldsymbol{u}}{\partial t}+(\boldsymbol{u} \cdot \nabla) \boldsymbol{u}=-\nabla p+\nu \nabla^{2} \boldsymbol{u}+\frac{\boldsymbol{J} \times \boldsymbol{B}}{\rho} \\
& \boldsymbol{B}(\boldsymbol{r})=\frac{\mu_{0}}{4 \pi} \int \frac{\boldsymbol{J}\left(\boldsymbol{r}^{\prime}\right) \times\left(\boldsymbol{r}-\boldsymbol{r}^{\prime}\right)}{\left|\boldsymbol{r}-\boldsymbol{r}^{\prime}\right|^{3}} d V^{\prime}
\end{aligned}
$$

with $t, \boldsymbol{u}, p, \nu, \rho, \mu_{0}, \boldsymbol{r}$ and $d V$ denoting the time, the velocity, the pressure, the kinematic viscosity, the density, the vacuum permeability, the coordinate and the differential volume, respectively. The fluid mesh has at least 200 cells on the diameter, which is fine enough according to [43].

Thermal effects are modelled in the fluid only using the Oberbeck-Boussinesq approximation [80] (for its validity, see [43, 81]). The following set of equations 
is solved

$$
\begin{aligned}
\frac{\partial \boldsymbol{u}}{\partial t}+\nabla \cdot(\boldsymbol{u u}) & =-\nabla p_{d}+\nu \nabla^{2} \boldsymbol{u}-\boldsymbol{g} \cdot \boldsymbol{r} \nabla \rho_{k}+\frac{\boldsymbol{J} \times \boldsymbol{B}}{\rho_{0}} \\
\nabla \cdot \boldsymbol{u} & =0 \\
\frac{\partial T}{\partial t}+\nabla \cdot(\boldsymbol{u} T) & =\frac{\lambda}{\rho_{0} c_{p}} \nabla^{2} T
\end{aligned}
$$

with $\boldsymbol{u}, p, \nu, \boldsymbol{g}, \boldsymbol{r}, T, c_{p} \boldsymbol{J}$ and $\sigma$ denoting velocity, pressure, kinematic viscosity, gravity, position vector, temperature, specific heat capacity, current density and electrical conductivity, respectively. The density $\rho=\rho_{0} \rho_{k}=\rho_{0}\left(1-\beta\left(T-T_{\text {ref }}\right)\right)$ is calculated using the mean density $\rho_{0}$ at reference temperature $T_{\text {ref }}$ and the coefficient of thermal expansion $\beta . \quad \boldsymbol{J}$ and $\boldsymbol{B}$ are determined by the electrovortex solver as described above; the resulting Lorenz force is assumed to be constant in time, i.e. induced currents are neglected. At least 250 cells on the diameter and strongly refined boundary layers are used. No-slip boundary conditions are used for velocity. This is justified even for the free surface due to the oxide film formed there 82 . The side walls are modelled as adiabatic while a constant temperature is applied at the top and bottom boundaries.

\section{Results}

This section is arranged as follows: firstly, we compare the influence of a symmetric and asymmetric current supply on pure electro-vortex flow (fig. 4). Thereafter, we study the influence of the Earth magnetic field and of thermal stratification on both connection types (fig. 5 and 6). Further, we give estimates of the flow velocity depending on the cell current.

Fig. 4a illustrates the current path, streamlines and velocities for a lateral supply line. Electro-vortex flow is simulated alone; the applied current is $40 \mathrm{~A}$. The flow profile is essentially horizontal forming two kidney-shaped vortices. The velocity reaches $2.5 \mathrm{~mm} / \mathrm{s}$. The horizontal jet (also shown in 4 4 ) is uncommon for electro-vortex flow, but can easily be explained. As the current flows mainly horizontally through the copper plate, it induces a magnetic field in the fluid. This field points towards the observer (in fig. 4 a and c). The current in 

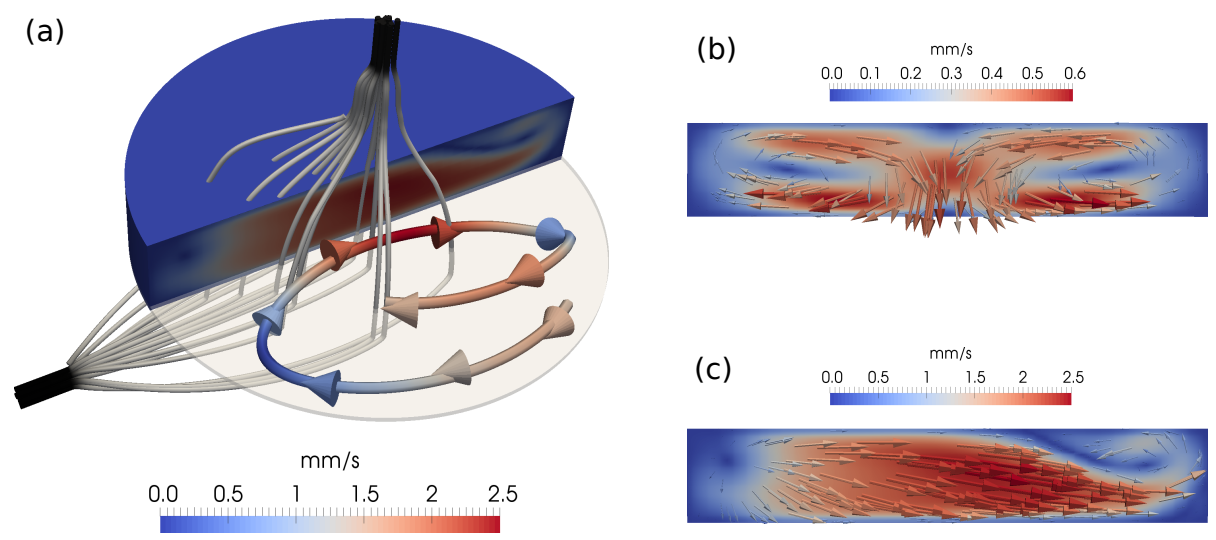

Figure 4: Current path and velocity streamlines for a current supply from the side (a). Velocity on a vertical plane for symmetric (b) and lateral current supply (c). The current is $I=40 \mathrm{~A}$; the results show electro-vortex flow alone.

the liquid metal flows upwards (vertically) and interacts with the induced field. Consequently, the Lorentz force points to the right and drives the observed flow in "prolongation" of the current supply. For similar experiments, see [83, 84].

Fig. $4 \mathrm{p}$ shows the flow for a symmetric current supply, again at $40 \mathrm{~A}$. A typical poloidal flow develops as it was often observed experimentally [47, 8589. Similar flow structures are very well known from vacuum arc remelting and electro-slag remelting [90 97. However, depending on the exact geometry, the direction of the flow might be reversed [98-101]. In our simulation, the velocities reach $0.6 \mathrm{~mm} / \mathrm{s}$ for the symmetrical setup. This is only $25 \%$ of the flow velocity observed for a lateral current supply. Due to the shallow liquid metal layer, a poloidal flow will dissipate strongly in the boundary layer.

The simulated velocities are not directly comparable to the experiment 64]. The latter was additionally heated from below (vertical temperature difference of approximately $\Delta T=10 \mathrm{~K})$. As shown numerically by Beltrán, the experimentally observed flow is mainly caused by Rayleigh-Bénard convection. Also he used the linear expansion coefficient [64, 102] which is three times smaller than the volumetric one [43], his velocity profile and magnitude $(3 \mathrm{~mm} / \mathrm{s})$ matches very well to the experimental results (compare fig. 9 in 102 and fig. 4 in 
64]). Electro-vortex flow will generally lead to velocities one order of magnitude smaller (Kelley and Sadoway [64] used currents of 23.3 A at most; our results are for $40 \mathrm{~A}$ ). However, electro-vortex flow will surely influence the flow structure and may explain the increase in order of the flow which was observed experimentally.

In the next step we focus on the symmetric current supply (with the poloidal flow) only, and analyse the influence of a vertical magnetic background field. When we add the magnetic field of the Earth (measured in Dresden as $\boldsymbol{B}=$ $\left(15 \cdot \boldsymbol{e}_{x}, 5 \cdot \boldsymbol{e}_{y}, 36 \cdot \boldsymbol{e}_{z}\right) \mu \mathrm{T}$ ), the original poloidal flow (fig. 5 a) becomes strongly helical (fig. 5 b). The appearance of such azimuthal swirl flow is well known from experiments [43, 85, 103] and can be easily explained. Radial cell currents and a vertical magnetic background field lead to azimuthal Lorentz forces [87, 92, 103]. Compared to a recent experiment by Ashour et al. 443 with a point electrode on the top, we observe considerably stronger swirl (compare fig. 5 b with fig. 5 in [43]). We attribute this difference to the location of the azimuthal forcing. Here, the force is well distributed in the whole volume; in 43 it is concentrated only in the centre of the liquid metal "sheet". We suppose the distributed azimuthal Lorentz force to better suppress the poloidal flow by forcing the streamlines into a dissipative Ekman layer 92. Fig. 5s shows the volume averaged mean velocity of the poloidal and azimuthal flow - with and without the Earth magnetic field. If we add a vertical field, azimuthal swirl appears (compare the dashed curve). At the same time, the poloidal flow is strongly reduced (by a factor of $1 / 2$ ). This fits nicely to Davidsons "poloidal suppression" model 92. This is remarkable, because simulations with a point electrode (see [43]) did not show such a strong suppression.

Keeping the symmetric current supply, we now focus on the influence of the thermal stratification. During operation of an LMB, the cathode will be heated from above; the thermal stratification will be stable. At first glance, this configuration is similar to arc remelting. There, an electric arc heats the melt from above. However, the bath is cooled rather from the side than from below which leads to strong thermally driven flow [104, but we have a stable thermal 

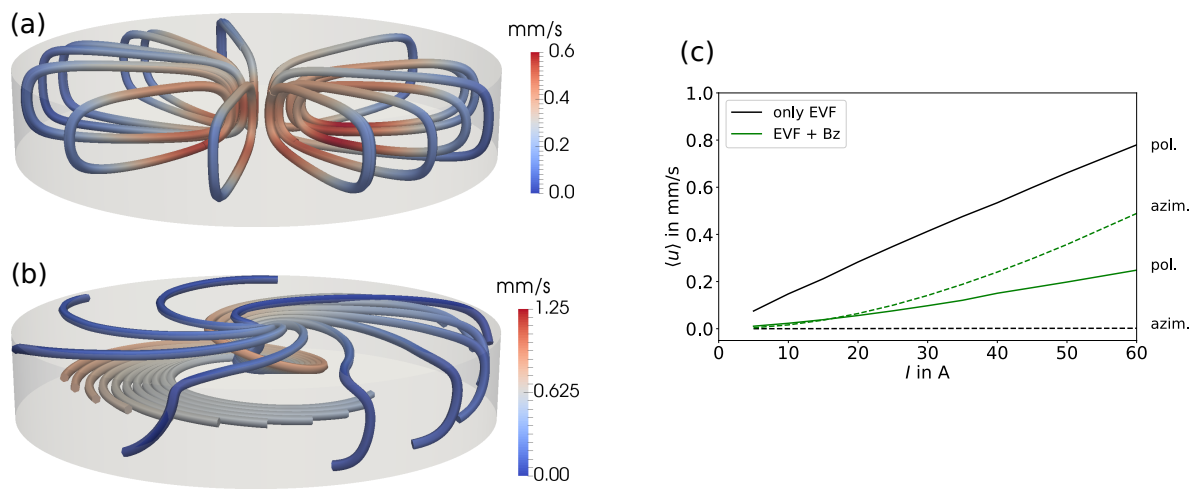

(d)

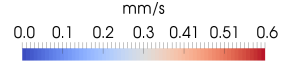

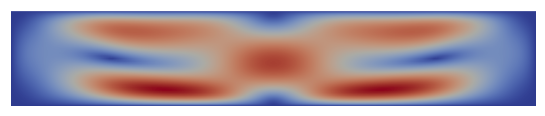

(f)
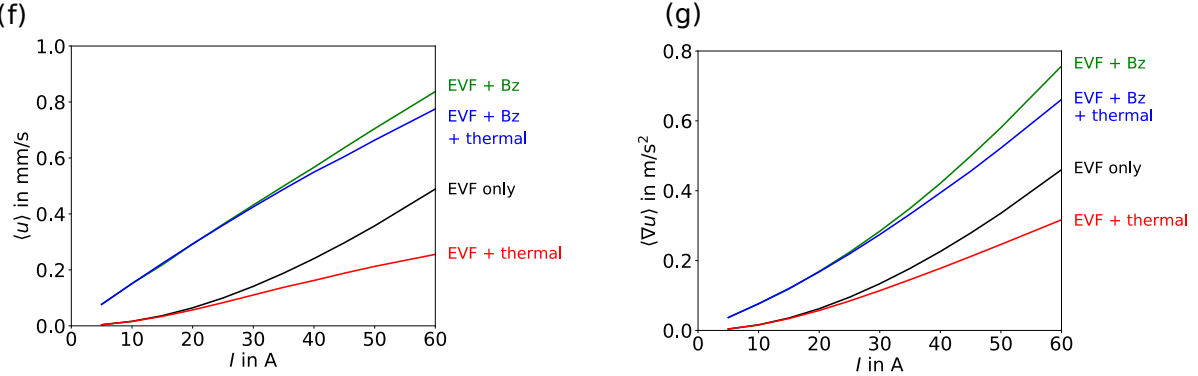

Figure 5: Streamlines and velocity without (a) and with the Earth magnetic field (b). Volume averaged mean velocities of the azimuthal and poloidal flow for both cases (c). Velocity on a vertical plane for symmetric current supply without temperature (d) and with a negative temperature gradient of $0.7 \mathrm{~K}(\mathrm{e})$. Volume averaged mean velocity (f) and mean velocity gradient evaluated at the top surface $(\mathrm{g})$ of electro-vortex flow alone, with an additional Earth magnetic field $(\mathrm{Bz})$ and with a stabilising temperature gradient. $I=40 \mathrm{~A}$.

stratification instead. Based on the heat conduction model described in section 2 we apply a vertical temperature gradient of $\Delta T=0.7 \mathrm{~K}$ (at $40 \mathrm{~A}$ ). The stable thermal stratification dampens the electro-vortex flow (compare fig. $5 \mathrm{~d}$ and e). While the general flow structure does not change, especially the velocity near the bottom wall decreases by a factor of $2 / 3$. This result cannot be compared to the experiment, as Kelley and Sadoway heated from below (and we from above). 
A temperature gradient as in the experiment is not expected to appear during "normal" operation of an LMB; however, an additional heating or cooling for mass transfer enhancement (as proposed in [39, 40]) can easily lead to similar configurations.

Using a thermal diffusivity of $\alpha=6 \cdot 10^{-6} \mathrm{~m}^{2} / \mathrm{s}$, a mass diffusivity of $D=$ $1.2 \cdot 10^{-8} \mathrm{~m}^{2} / \mathrm{s}$ [6], a typical velocity scale of $u=1 \mathrm{~mm} / \mathrm{s}$ and the height of the liquid metal $\Delta h=16 \mathrm{~mm}$, we find a thermal Peclet number of $\mathrm{Pe}_{\mathrm{th}}=$ $u \Delta h / \alpha \approx 5$ and a concentration Peclet number of $\mathrm{Pe}_{\mathrm{c}}=u \Delta h / D \approx 6000$ 105]. Obviously, convection dominates mass transfer. We use thererfore two quantities to estimate mixing in the cathode: the volume averaged velocity as global measure, and the mean velocity gradient at the foam-cathode interface as local one. Fig. 5f and g show both quantities for electro-vortex flow alone, with the Earth magnetic field ("Bz") and with a stabilising thermal gradient. The azimuthal flow, caused by the Earth magnetic field, yields the highest velocities. A vertical temperature gradient does barely influence the horizontal flow. The poloidal electro-vortex flow ("EVF alone") is considerably slower it is strongly dissipated at the bottom wall. The vertical temperature gradient effectively breaks the downwards flow. Interestingly, a strong flow in the volume leads also to strong velocity gradients at the interface.

We now consider the lateral current supply, and study again the influence of temperature and the Earth magnetic field. The prevailing horizontal flow is hardly influenced by a stabilising vertical temperature gradient. The flow structure changes only slightly; the velocities with and without temperature gradient are almost the same (compare fig. 6e and d). Taking into account the Earth magnetic field changes the flow much more (compare fig. $6 \mathrm{a}$ and b). The horizontal current and vertical magnetic background field generate a Lorentz force which deflects the jet in clockwise direction. Presumably the stronger dissipation in the boundary layers decreases the velocity slightly. Most importantly, the Earth magnetic field does not lead to swirl flow in this configuration - the jet is only deflected. Fig. 6e and $\mathrm{f}$ show the mean velocity and the mean velocity gradient for pure electro-vortex flow, with the Earth magnetic field and with 
the stabilising temperature gradient. The differences are only marginal.
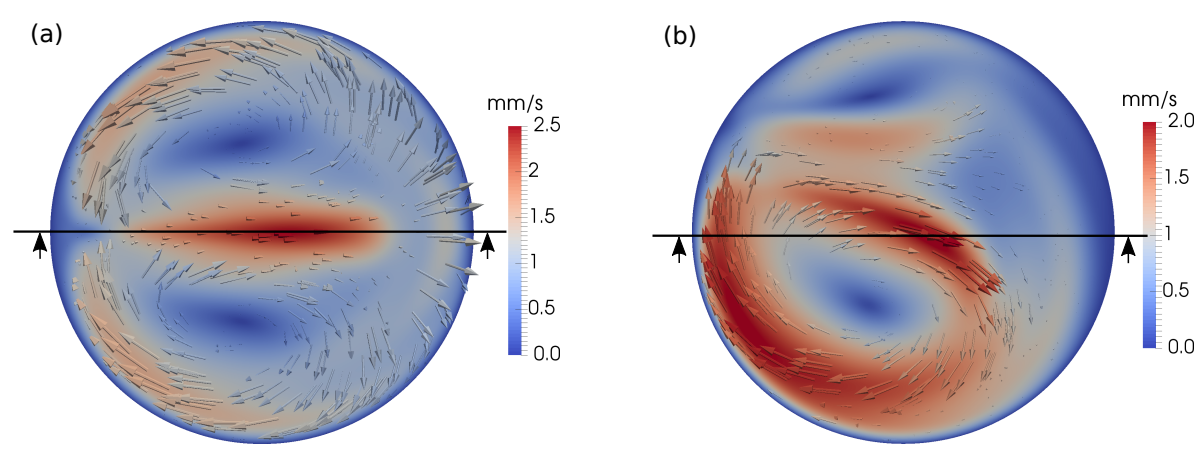

(c)

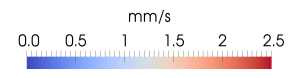

(d)
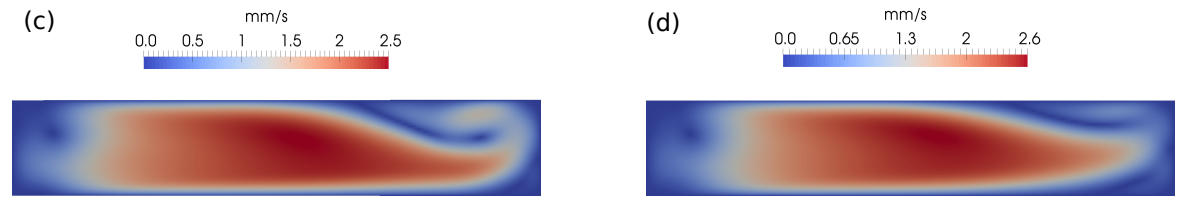

(e)
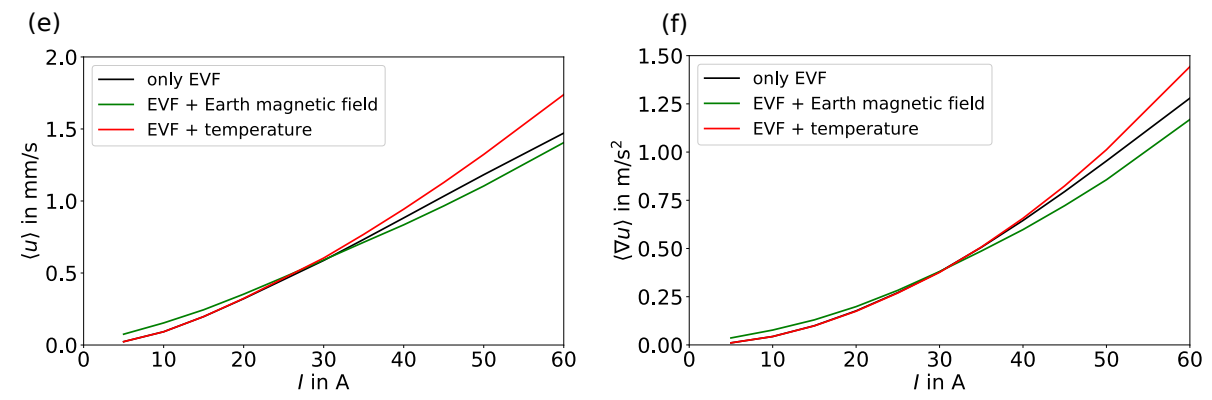

Figure 6: Electro-vortex flow for a lateral supply wire without (a) and with the Earth magnetic field (b). Flow in the cross section of the jet without (c) and with a stabilising thermal gradient (d). The current for (a)-(d) is $40 \mathrm{~A}$. Volume averaged mean velocity (e) and mean velocity gradient (f) for electro-vortex flow alone, with the additional Earth magnetic field and with a stabilising temperature gradient.

\section{Summary \& outlook}

We have shown, how electro-vortex flow (EVF) has the potential to enhance mass transfer in liquid metal batteries (LMBs). In a first step we discussed why such mass transfer enhancement is important. Considering the high concentration Peclet number (in the order of $10^{3}$ ), we pointed out that convection 
(and not diffusion) will dominate mass transfer. We further emphasised that mostly (but not only) mixing of the positive electrode during discharge is highly beneficial. We studied the flow structure and magnitude of EVF numerically. Moreover, we discussed the influence of stray magnetic fields, the connection of the supply lines and a stable thermal stratification on electro-vortex flow.

A lateral current supply to the cathode will generate a horizontal flow. In contrast, a centrical current supply below the cathode will induce a vertical jet. Looking only on this flow-direction, we would expect a vertical flow to be better suited for enhancing mass transfer. It will remove reaction products directly from the cathode-electrolyte interface. However, the vertical (or better: poloidal) flow has three disadvantages: (1) it's mean velocity is much smaller compared to the horizontal flow, (2) it is dampened by the stable thermal stratification and (3) it will turn to a swirling flow under presence of the Earth magnetic field. In contrast, the horizontal jet will not be dampened considerably by a thermal stratification nor be strongly influenced by the Earth magnetic field. We believe therefore the lateral supply line to be better suited for enhancing mass transfer. Concerning the swirl flow we could (at least partially) confirm Davidsons model of poloidal suppression.

Our models are strongly simplified: we ignore induced currents and magnetic fields; the simulation of thermal convection and EVF is decoupled. A next step would be therefore the development of a fully coupled EVF-thermal convection model as well as it's coupling with a real mass transfer (e.g. Li in Bi) model. Of course, velocity and concentration measurements in a real 3-layer LMB would be a large step forward. Performing Kelley \& Sadoway's experiment with an inverse temperature gradient could allow a further experimental study of the interaction between EVF and thermal convection. Such an experiment should preferably conducted at room temperature to ensure well defined boundary conditions for temperature. 


\section{Acknowledgements}

This work was supported by the Deutsche Forschungsgemeinschaft (DFG, German Research Foundation) by award number 338560565 as well as the Helmholtz-Gemeinschaft Deutscher Forschungszentren (HGF) in frame of the Helmholtz Alliance "Liquid metal technologies" (LIMTECH). The computations were performed on the Bull HPC-Cluster "Taurus" at the Centre for Information Services and High Performance Computing (ZIH) at TU Dresden and on the cluster "Hydra" at Helmholtz-Zentrum Dresden - Rossendorf. Fruitful discussions with V. Bojarevics, P. Davidson, D. Kelley, F. Stefani and T. Vogt on several aspects of electro-vortex flow and thermal convection are gratefully acknowledged. N. Weber thanks Henrik Schulz for the HPC support.

\section{References}

\section{References}

[1] E. J. Cairns, C. E. Crouthamel, A. K. Fischer, M. S. Foster, J. C. Hesson, C. E. Johnson, H. Shimotake, A. D. Tevebaugh, Galvanic Cells with Fused-Salt Electrolytes, ANL-7316, Argonne National Laboratory, 1967.

[2] H. L. Chum, R. A. Osteryoung, Review of Thermally Regenerative Electrochemical Systems, Solar Energy Research Institute, 1980.

[3] H. L. Chum, R. A. Osteryoung, Review of Thermally Regenerative Electrochemical Cells, Solar Energy Research Institute, 1981.

[4] D. A. J. Swinkels, Molten Salt Batteries and Fuel Cells, in: J. Braunstein, G. Mamantov, G. P. Smith (Eds.), Advances in Molten Salt Chemistry, Vol. 1, Plenum Press, New York, 1971, pp. 165-223.

[5] H. Kim, D. A. Boysen, J. M. Newhouse, B. L. Spatocco, B. Chung, P. J. Burke, D. J. Bradwell, K. Jiang, A. A. Tomaszowska, K. Wang, W. Wei, L. A. Ortiz, S. A. Barriga, S. M. Poizeau, D. R. Sadoway, Liquid Metal 
Batteries: Past, Present, and Future, Chem. Rev. 113 (3) (2013) 20752099. doi:10.1021/cr300205k.

[6] D. H. Kelley, T. Weier, Fluid mechanics of liquid metal batteries, Appl. Mech. Rev. 70 (2) (2018) 020801. doi:10.1115/1.4038699.

[7] H. Kim, D. A. Boysen, T. Ouchi, D. R. Sadoway, Calcium - bismuth electrodes for large - scale energy storage, J. Power Sources 241 (2013) 239-248.

[8] T. Ouchi, H. Kim, B. L. Spatocco, D. R. Sadoway, Calcium-based multielement chemistry for grid-scale electrochemical energy storage, Nat. Commun. 7 (2016) 10999. doi:10.1038/ncomms10999.

[9] S. Poizeau, H. Kim, J. M. Newhouse, B. L. Spatocco, D. R. Sadoway, Determination and modeling of the thermodynamic properties of liquid calcium-antimony alloys, Electrochimica Acta 76 (2012) 8-15. doi:10. $1016 / \mathrm{j}$.electacta.2012.04.139

[10] B. Agruss, H. R. Karas, First Quarterly Technical Progress Report on Design and Development of a Liquid Metal Cell for the Period 1 January 1962 - 31 March 1962, Tech. Rep. AD 274 197, Armed Service Technical Information Agency (1962).

[11] B. Agruss, H. R. Karas, The Thermally Regenerative Liquid Metal Concentration Cell, in: R. F. Gold (Ed.), Regenerative EMF Cells, Vol. 64 of Advances in Chemistry, American Chemical Society, Washington, D.C., 1967, pp. 62-81.

[12] S. Lawroski, R. C. Vogel, M. Levenson, V. H. Munnecke, Chemical engineering summary report, Tech. Rep. ANL-6648, Argonne National Laboratory (1963).

[13] M. S. Foster, S. E. Wood, C. E. Crouthamel, Thermodynamics of Binary Alloys. I. The Lithium-Bismuth System, Inorg. Chem. 3 (10) (1964) 14281431. 
[14] R. C. Vogel, M. Levenson, J. H. Schraidt, J. Royal, Chemical engineering divison research highlights, Tech. Rep. ANL-7175, Argonne National Laboratory (1966).

[15] H. Shimotake, G. L. Rogers, E. J. Cairns, Secondary Cells with Lithium Anodes and Immobilized Fused-Salt Electrolytes, Ind. Eng. Chem. Process Des. Dev. 8 (1) (1969) 51-56.

[16] X. Ning, S. Phadke, B. Chung, H. Yin, P. Burke, D. R. Sadoway, Selfhealing Li-Bi liquid metal battery for grid-scale energy storage, J. Power Sources 275 (2015) 370-376. doi:10.1016/j.jpowsour.2014.10.173.

[17] K. Wang, K. Jiang, B. Chung, T. Ouchi, P. J. Burke, D. A. Boysen, D. J. Bradwell, H. Kim, U. Muecke, D. R. Sadoway, Lithium-antimony-lead liquid metal battery for grid-level energy storage, Nature 514 (7522) (2014) 348-350. doi:10.1038/nature13700.

[18] R. C. Vogel, M. Levenson, F. R. Masten, Chemical engineering division research highlights, Tech. Rep. ANL-7020, Argonne National Laboratory (1965).

[19] M. S. Foster, C. E. Crouthamel, S. E. Wood, Thermodynamics of Binary Alloys. II. The Lithium-Tin System, J. Phys. Chem. 70 (10) (1966) 30423045 .

[20] J. C. Hesson, H. Shimotake, Thermodynamics and thermal efficiencies of thermally regenerative bimetallic and hydride emf cell systems, in: Regenerative EMF Cells, American Chemical Society, 1967, pp. 82-104.

[21] D. J. Bradwell, Liquid Metal Batteries: Ambipolar Electrolysis and Alkaline Earth Electroalloying Cells, Ph.D. thesis, Massachusetts Institute of Technology (2011).

[22] D. J. Bradwell, H. Kim, A. H. C. Sirk, D. R. Sadoway, Magnesium-Antimony Liquid Metal Battery for Stationary Energy Storage, J. Am. Chem. Soc. 134 (4) (2012) 1895-1897. doi:10.1021/ja209759s. 
[23] M. S. Foster, G. H. McCloud, E. J. Cairns, Electrochemical studies of the Sodium-Bismuth system, J. Am. Chem. Soc. (1967) 276-281.

[24] R. C. Vogel, M. Levenson, E. R. Proud, J. Royal, Chemical engineering division research highlights, Tech. Rep. ANL-7350, Argonne National Laboratory (1967).

[25] R. C. Vogel, M. Levenson, F. R. Masten, Chemical engineering divison semiannual report, Tech. Rep. ANL-7055, Argonne National Laboratory (1965).

[26] H. Shimotake, E. J. Cairns, Bimetallic galvanic cells with fused-salt electrolytes, in: Advances in Energy Conversion Engineering, ASME, Florida, 1967, pp. 951-962.

[27] R. C. Vogel, M. Levenson, J. H. Schraidt, J. Royal, Chemical engineering semiannual report, Tech. Rep. ANL-7225, Argonne National Laboratory (1966).

[28] L. A. Herédy, M. L. Iverson, G. D. Ulrich, H. L. Recht, Development of a thermally regenerative Sodium-Mercury galvanic system Part I Electrochemical and Chemical Behavior of Sodium-Mercury Galvanic Cells, in: Regenerative EMF Cells, American Chemical Society, 1967, pp. 30-42.

[29] B. L. Spatocco, P. J. Burke, D. R. Sadoway, Low temperature liquid metal batteries for grid-scaled storage, 0099522 A1 (2014).

[30] A. Kisza, J. Kazmierczak, B. Borresen, G. M. Haarberg, R. Tunold, The Kinetics of the Sodium Electrode Reaction in Molten Sodium Chloride, J. Electrochem. Soc. 142 (4) (1995) 1035-1040.

[31] R. D. Weaver, S. W. Smith, N. L. Willmann, The Sodium-Tin LiquidMetal Cell, J. Electrochem. Soc. 109 (8) (1962) 653-657.

[32] B. Agruss, The Thermally Regenerative Liquid-Metal Cell, J. Electrochem. Soc. 110 (11) (1963) 1097-1103. 
[33] J. Xu, O. S. Kjos, K. S. Osen, A. M. Martinez, O. E. Kongstein, G. M. Haarberg, Na-Zn liquid metal battery, J. Power Sources 332 (2016) 274280. doi:10.1016/j.jpowsour.2016.09.125.

[34] J. Xu, A. M. Martinez, K. S. Osen, O. S. Kjos, O. E. Kongstein, G. M. Haarberg, Electrode Behaviors of Na-Zn Liquid Metal Battery, J. Electrochem. Soc. 164 (12) (2017) A2335-A2340.

[35] E. J. Cairns, H. Shimotake, High-Temperature Batteries, Science 164 (1969) 1347-1355.

[36] E. J. Cairns, H. Shimotake, Recent advances in fuel cells and their application to new hybrid systems, Adv. Chem. 90 (1969) 321-350.

[37] M. S. Foster, Laboratory Studies of Intermetallic Cells, in: Regenerative EMF Cells, American Chemical Society, 1967, pp. 136-148.

[38] T. Ouchi, H. Kim, X. Ning, D. R. Sadoway, Calcium-Antimony Alloys as Electrodes for Liquid Metal Batteries, J. Electrochem. Soc. 161 (12) (2014) A1898-A1904.

[39] D. Bradwell, G. Ceder, L. A. Ortiz, D. R. Sadoway, Liquid electrode battery, US patent 2011/0014505 A1 (2011).

[40] D. Bradwell, G. Ceder, L. A. Ortiz, D. R. Sadoway, Liquid metal alloy energy storage device, US patent 9,076,996 B2 (2015).

[41] N. Weber, V. Galindo, J. Priede, F. Stefani, T. Weier, The influence of current collectors on Tayler instability and electro vortex flows in liquid metal batteries, Phys. Fluids 27 (2015) 014103.

[42] F. Stefani, V. Galindo, C. Kasprzyk, S. Landgraf, M. Seilmayer, M. Starace, N. Weber, T. Weier, Magnetohydrodynamic effects in liquid metal batteries, IOP Conf. Ser. Mater. Sci. Eng. 143 (2016) 012024. doi:10.1088/1757-899X/143/1/012024. 
[43] R. Ashour, D. H. Kelley, A. Salas, M. Starace, N. Weber, T. Weier, Competing forces in liquid metal electrodes and batteries, J. Power Sources 378 (2018) 301-310. doi:10.1016/j.jpowsour.2017.12.042

[44] S. Lundquist, On the hydromagnetic viscous flow generated by a diverging electric current, Ark. För Fys. 40 (5) (1969) 89-95.

[45] J. A. Shercliff, Fluid motions due to an electric current source, J. Fluid Mech. 40 (1970) 241-250.

[46] P. A. Davidson, An Introduction to Magnetohydrodynamics, Cambridge texts in applied mathematics, Cambridge University Press, Cambridge ; New York, 2001.

[47] V. Bojarevičs, Y. Freibergs, E. I. Shilova, E. V. Shcherbinin, Electrically Induced Vortical Flows, Kluwer Academic Publishers, 1989.

[48] Y. V. Vandakurov, Theory for the stability of a star with a toroidal magnetic field, Astron Zh 49 (2) (1972) 324-333.

[49] F. Stefani, T. Weier, T. Gundrum, G. Gerbeth, How to circumvent the size limitation of liquid metal batteries due to the Tayler instability, Energy Convers. Manag. 52 (2011) 2982-2986.

[50] M. Seilmayer, F. Stefani, T. Gundrum, T. Weier, G. Gerbeth, M. Gellert, G. Rüdiger, Experimental Evidence for a Transient Tayler Instability in a Cylindrical Liquid-Metal Column, Phys. Rev. Lett. 108 (2012) 244501.

[51] N. Weber, V. Galindo, F. Stefani, T. Weier, T. Wondrak, Numerical simulation of the Tayler instability in liquid metals, New J. Phys. 15 (2013) 043034 .

[52] N. Weber, V. Galindo, F. Stefani, T. Weier, Current-driven flow instabilities in large-scale liquid metal batteries, and how to tame them, J. Power Sources 265 (2014) 166-173. doi:10.1016/j.jpowsour.2014.03.055. 
[53] N. Weber, V. Galindo, F. Stefani, T. Weier, The Tayler instability at low magnetic Prandtl numbers: Between chiral symmetry breaking and helicity oscillations, New J. Phys. 17 (11) (2015) 113013. doi:10.1088/ $1367-2630 / 17 / 11 / 113013$.

[54] W. Herreman, C. Nore, L. Cappanera, J.-L. Guermond, Tayler instability in liquid metal columns and liquid metal batteries, J. Fluid Mech. 771 (2015) 79-114. doi:10.1017/jfm.2015.159.

[55] F. Stefani, V. Galindo, A. Giesecke, N. Weber, T. Weier, The Tayler instability at low magnetic Prandtl numbers: Chiral symmetry breaking and synchronizable helicity oscillations, Magnetohydrodynamics 53 (1) (2017) 169-178.

[56] T. Weier, A. Bund, W. El-Mofid, G. M. Horstmann, C.-C. Lalau, S. Landgraf, M. Nimtz, M. Starace, F. Stefani, N. Weber, Liquid metal batteries - materials selection and fluid dynamics, IOP Conf. Ser. Mater. Sci. Eng. 228 (2017) 012013.

[57] Y. Shen, O. Zikanov, Thermal convection in a liquid metal battery, Theor. Comput. Fluid Dyn. 30 (4) (2016) 275-294. doi:10.1007/ s00162-015-0378-1.

[58] T. Köllner, T. Boeck, J. Schumacher, Thermal Rayleigh-Marangoni convection in a three-layer liquid-metal-battery model, Phys. Rev. E 95 (2017) 053114. doi:10.1103/PhysRevE.95.053114

[59] O. Zikanov, Metal pad instabilities in liquid metal batteries, Phys. Rev. E 92 (2015) 063021.

[60] N. Weber, P. Beckstein, V. Galindo, W. Herreman, C. Nore, F. Stefani, T. Weier, Metal pad roll instability in liquid metal batteries, Magnetohydrodynamics 53 (1) (2017) 129-140.

[61] N. Weber, P. Beckstein, W. Herreman, G. M. Horstmann, C. Nore, F. Stefani, T. Weier, Sloshing instability and electrolyte layer rupture 
in liquid metal batteries, Phys. Fluids 29 (5) (2017) 054101. doi: $10.1063 / 1.4982900$

[62] V. Bojarevics, A. Tucs, MHD of Large Scale Liquid Metal Batteries, in: A. P. Ratvik (Ed.), Light Metals 2017, Springer International Publishing, Cham, 2017, pp. 687-692.

[63] G. M. Horstmann, N. Weber, T. Weier, Coupling and stability of interfacial waves in liquid metal batteries, ArXiv Prepr. ArXiv170802159.

[64] D. H. Kelley, D. R. Sadoway, Mixing in a liquid metal electrode, Phys. Fluids 26 (5) (2014) 057102. doi:10.1063/1.4875815.

[65] V. Sobolev, Thermophysical properties of lead and lead-bismuth eutectic, J. Nucl. Mater. 362 (2-3) (2007) 235-247. doi:10.1016/j.jnucmat. 2007.01 .144

[66] V. Sobolev, Database of Thermophysical Properties of Liquid Metal Coolants for GEN-IV, SCK CEN, 2010.

[67] N. E. Agency, Handbook on Lead-bismuth Eutectic Alloy and Lead Properties, Materials Compatibility, Thermalhydraulics and Technologies, Tech. Rep. 7268, Nuclear Energy Agency (2015).

[68] K. P. Dharmasena, H. N. G. Wadley, Electrical Conductivity of Open-cell Metal Foams, J. Mater. Res. 17 (03) (2002) 625-631. doi:10.1557/JMR. 2002.0089

[69] X. Huang, G. Wu, Z. Lv, Q. Zhang, P. Kang, J. Leng, Electrical conductivity of open-cell Fe-Ni alloy foams, J. Alloys Compd. 479 (1-2) (2009) 898-901. doi:10.1016/j.jallcom.2009.01.099.

[70] P. Personnettaz, Assessment of thermal phenomena in Li||Bi liquid metal batteries through analytical and numerical models, master thesis, Politecnico di Torino (2017). 
[71] W. Wang, K. Wang, Simulation of thermal properties of the liquid metal batteries, in: 6th International Conference on Power Electronics Systems and Applications (PESA), IEEE, 2015, pp. 1-11.

[72] N. Weber, Modellierung von Tayler-Instabilität und Elektrowirbelströmungen in Flüssigmetallbatterien, Ph.D. thesis, Technische Universität Dresden (2016).

[73] R. W. Ohse, et al., Handbook of thermodynamic and transport properties of alkali metals, Blackwell Oxford, 1985.

[74] G. J. Janz, C. B. Allen, N. P. Bansal, R. M. Murphy, R. P. T. Tomkins, Physical Properties Data Compilations Relevant to Energy Storage. II. Molten Salts: Data on Single and Multi-Component Salt Systems, U. S. Department of Commerce, 1979.

[75] G. J. Janz, Thermodynamic and Transport Properties for Molten Salts: Correlation Equations for Critically Evaluated Density, Surface Tension, Electrical Conductance, and Viscosity Data, no. v. 17 in Journal of physical and chemical reference data: Supplement, American Chemical Society and the American Institute of Physics, 1988.

URL https://books .google.de/books?id=qSXxAAAAMAAJ

[76] C. Fazio, V. Sobolev, A. Aerts, S. Gavrilov, K. Lambrinou, P. Schuurmans, A. Gessi, P. Agostini, A. Ciampichetti, L. Martinelli, et al., Handbook on lead-bismuth eutectic alloy and lead properties, materials compatibility, thermal-hydraulics and technologies., Tech. rep., Organisation for Economic Co-Operation and Development (2015).

[77] K. Cornwell, The thermal conductivity of molten salts, J. Phys. Appl. Phys. 4 (3) (1971) 441.

[78] H. G. Weller, G. Tabor, H. Jasak, C. Fureby, A tensorial approach to computational continuum mechanics using object-oriented techniques, Comput. Phys. 12 (6) (1998) 620-631. 
[79] N. Weber, P. Beckstein, V. Galindo, M. Starace, T. Weier, Electro-vortex flow simulation using coupled meshes, ArXiv Prepr. ArXiv170706546.

[80] A. Oberbeck, Über die Wärmeleitung der Flüssigkeiten bei der Berücksichtigung der Strömungen infolge von Temperaturdifferenzen, Ann. Phys. Chem. 7 (271).

[81] D. D. Gray, A. Giorgini, The validity of the Boussinesq approximation for liquids and gases, Int. J. Heat Mass Transf. 19 (5) (1976) 545-551.

[82] A. Cramer, J. Pal, G. Gerbeth, Ultrasonic flow measurements in a model of a Czochralski puller, Flow Meas. Instrum. 37 (2014) 99-106. doi: 10.1016/j.flowmeasinst.2014.03.008

[83] S. B. Dement'ev, O. M. Skopis, E. V. Sherbinin, Intensification of the mixing process in direct-current electric arc furnaces, Magnetohydrodynamics 28 (1) (1992) 89-92.

[84] I. Kolesnichenko, S. Khripchenko, D. Buchenau, G. Gerbeth, Electrovortex flows in a square layer of liquid metal, Magnetohydrodynamics 41 (2005) 39-51.

[85] R. A. Woods, D. R. Milner, Motion in the weld pool in arc welding, Weld. J. 50 (1971) 163-173.

[86] I. E. Butsenieks, D. E. Peterson, V. I. Sharamkin, E. V. Sherbinin, Magnetohydrodynamic fluid flows in a closed space with a nonuniform electric current, Magn. Gidrodin. 1 (1976) 92-97.

[87] V. V. Boyarevich, V. I. Sharamkin, MHD flows due to current spreading in an axisymmetric layer of finite thickness, Magn. Gidrodin. 2 (1977) $55-60$.

[88] V. G. Zhilin, Y. P. Ivochkin, A. A. Oksman, G. R. Lurin'sh, A. I. Chaikovskii, A. Y. Chudnovskii, E. V. Shcherbinin, An experimental investigation of the velocity field in an axisymmetric electrovortical flow in a cylindrical container, Magn. Gidrodin. 3 (1986) 110-116. 
[89] D. Räbiger, Y. Zhang, V. Galindo, S. Franke, B. Willers, S. Eckert, The relevance of melt convection to grain refinement in $\mathrm{Al}-\mathrm{Si}$ alloys solidified under the impact of electric currents, Acta Mater. 79 (2014) 327-338. doi:10.1016/j.actamat.2014.07.037.

[90] D. R. Atthey, A mathematical model for fluid flow in a weld pool at high currents, J. Fluid Mech. 98 (4) (1980) 787-801.

[91] P. A. Davidson, S. C. Flood, Natural convection in an aluminum ingot: A mathematical model, Metall. Mater. Trans. B 25 (2) (1994) 293-302.

[92] P. A. Davidson, D. Kinnear, R. J. Lingwood, D. J. Short, X. He, The role of Ekman pumping and the dominance of swirl in confined flows driven by Lorentz forces, Eur. J. Mech. - BFluids 18 (1999) 693-711.

[93] P. A. Davidson, Overcoming instabilities in aluminium reduction cells: A route to cheaper aluminium, Mater. Sci. Technol. 16 (5) (2000) 475-479.

[94] A. Kharicha, W. Schützenhöfer, A. Ludwig, R. Tanzer, M. Wu, On the importance of electric currents flowing directly into the mould during an ESR process, Steel Res. Int. 79 (8) (2008) 632-636.

[95] V. Shatrov, G. Gerbeth, Stability of the electrically induced flow between two hemispherical electrodes, Magnetohydrodynamics 48 (3) (2012) 469483.

[96] O. V. Kazak, A. N. Semko, Numerical modeling of electro-vortical flows in a confined volume, J. Eng. Phys. Thermophys. 85 (2012) 1167-1178.

[97] A. Kharicha, I. Teplyakov, Y. Ivochkin, M. Wu, A. Ludwig, A. Guseva, Experimental and numerical analysis of free surface deformation in an electrically driven flow, Exp. Therm. Fluid Sci. 62 (2015) 192-201. doi: 10.1016/j.expthermflusci.2014.11.014.

[98] O. Kazak, A. Semko, Modelling vortex fields in metal smelting furnaces, Int. J. Multiphysics 4 (4) (2010) 351-358. 
[99] O. V. Kazak, A. N. Semko, Electrovortex motion of a melt in DC furnaces with a bottom electrode, J. Eng. Phys. Thermophys. 84 (1) (2011) 223231.

[100] O. Kazak, Modeling of Vortex Flows in Direct Current (DC) Electric Arc Furnace with Different Bottom Electrode Positions, Metall. Mater. Trans. B 44 (5) (2013) 1243-1250. doi:10.1007/s11663-013-9899-4

[101] O. Semko, Y. Ivochkin, I. Teplyakov, K. O, Electro vortex flows in hemisphere volume with different bottom electrode positions, in: 9th PAMIR International Conference, Fundamental and Applied MHD, 2014.

[102] A. Beltrán, MHD natural convection flow in a liquid metal electrode, Appl. Therm. Eng. 114 (2016) 1203-1212. doi:10.1016/j.applthermaleng. 2016.09.006.

[103] V. Bojarevičs, E. V. Shcherbinin, Azimuthal rotation in the axisymmetric meridional flow due to an electric-current source, J. Fluid Mech. 126 (1983) $413-430$.

[104] P. Davidson, X. He, A. Lowe, Flow transitions in vacuum arc remelting, Mater. Sci. Technol. 16 (6) (2000) 699-711. doi:10.1179/ 026708300101508306 .

[105] W. M. Deen, Analysis of Transport Phenomena, Topics in chemical engineering, Oxford University Press, New York, 1998. 